\title{
A NEW APPROACH TO DEFORMATION ENERGY CALCULATIONS ${ }^{\star}$
}

\author{
C.M. KO, H.C. PAULI*, M. BRACK and G.E. BROWN \\ Department of Physics, SUNY at Stony Brook, L.I., N.Y. 11 790, USA
}

Received 11 June 1973

\begin{abstract}
A simplified microscopic method is used to calculate the deformation energy curves of heavy nuclei, which show a second minimun and two fission barriers at about the same deformations as in the shell-correction calculations.
\end{abstract}

Constrained Hartree-Fock (CHF) calculations of nuclear deformation energy curves with effective nucleon-nucleon interactions have been successfully applied to deformed heavy nuclei [1]. Such calculations still have some quantitative difficulties related to the choice of both the constraints and the parameters of the interaction. A less fundamental and simpler approach to the deformation energies is the shell-correction method proposed by Strutinsky [2]. This method has been widely used during the past five years and is able to explain many experimental data concerning the ground state properties of stable deformed nuclei as well as the properties of fissioning nuclei [3-5]. It has been shown numerically [6] that the shell-correction method is consistent with the HF theory to within about $\pm 1 \mathrm{MeV}$ in the calculated total binding energies.

In the present letter, we propose a method which makes use of the deformation parameters introduced in the (essentially macroscopic) shell-correction method. The success of such shell-correction calculations suggests that these deformation parameters may be useful in constructing a basis of single particle states for a simplified (i.e., not self-consistent) microscopic calculation of the deformation energy curve with an effective nucleon-nucleon interaction.

Starting with an effective Hamiltonian $H$, an upper bound on the total binding energy of a nucleus is given by $E=\langle\psi|H| \psi\rangle$. In usual HF theory, the ground state wave function $|\psi\rangle$ is assumed to be a Slater determinant of the single particle wave functions which are found by an iteration procedure based upon the variational principle. The deformation energy curve can

« Work supported in part by the U.S. Atomic Energy Commission.

* Permanent address: Institut für Theoretische Physik der Universität Basel, Schweiz. be obtained if appropriate constraints are included in the Hamiltonian. Our present approach is to relax the requirement of self-consistency and use only the Slater determinant constructed from the shell model wave functions $\phi_{i}^{\mathrm{s}}(r)$, which are the eigenfunctions of a deformed Woods-Saxon potential and are believed to be sufficiently close to the self-consistent wave functions. This potential was originally proposed by Damgaard et al. [7] and was used extensively in the shell-correction calculations $[3,8]$.

Encouraged by the recent CHF results with the Skyrme interaction $[1,9,10,14]$ we use this interaction in various parametrization. For comparison we also use the interaction proposed by Moszkowski [11, $12]$. Both interactions are modified $\delta$-interactions which differ only in their density dependence. Also, there is no p-wave component in the Moszkowski interaction. An advantage of these interactions is that the total binding energy of a nucleus can be written as an integral over an energy density, which is a simple algebraic functional of the local nuclear density $\rho(r)$ and its derivatives, the kinetic energy density $\tau(r)$, and a spin density $J(r)$. The integral is evaluated numerically by Gaussian quadratures. In constructing the densities, we have included pairing effects through the usual BCS equation. The Coulomb energy for the protons is calculated exactly for the direct term in all cases and approximately for the exchange term using the so-called Slater approximation [13] in the case of SKY SP (see below).

In the present calculations, we consider only axial and reflection symmetric deformations. The single particle wave functions $\phi_{i}^{\mathrm{s}}(r)$ are obtained by diagonalizing the shell model Hamiltonian in a truncated basis of deformed harmonic oscillator states $[7,8]$. A consequence of this truncation is that the total binding 
Table 1

Parameters for Skyrme and Moszkowski interactions

\begin{tabular}{|c|c|c|c|c|c|c|c|}
\hline & $t_{0}$ & $x_{0}$ & $t_{1}$ & $t_{2}$ & $t_{3}$ & $w_{0}$ & $t_{13}$ \\
\hline SKY I a) & -1057.3 & 0.56 & 235.9 & -100.0 & 14463.5 & 120.0 & \\
\hline SKY II ${ }^{\text {a) }}$ & -1169.9 & 0.34 & 585.6 & -27.1 & 9331.1 & 105.0 & \\
\hline SKY III b) & -1086.20 & 0.5175 & 341.14 & -120.0 & 13114.0 & 130.0 & 1982.9 \\
\hline SKY $\mathrm{X}^{\mathrm{c})}$ & -1133.4 & 0.49 & 395.0 & -95.0 & 14000.0 & 120.0 & \\
\hline \multirow[t]{2}{*}{ SKY XP ${ }^{d)}$} & -1128.8 & 0.45 & 395.0 & -95.0 & 14000.0 & 120.0 & \\
\hline & $\alpha$ & $\beta$ & $\gamma$ & & & & \\
\hline MDI II ${ }^{e)}$ & 1244.4 & 646.4 & 144.7 & & & & \\
\hline
\end{tabular}

a) SKY I and II as used by Vautherin and Brink $[9,10]$.

b) SKY III contains an additional momentum dependent three-body term, presently studied by Liu [16].

c) SKY $X$ proposed by Quentin et al. [14].

d) SKY XP same as SKY X, but including the exchange Coulomb energy in the fit of the parameters [15].

e) MDI II as used in ref. [12].

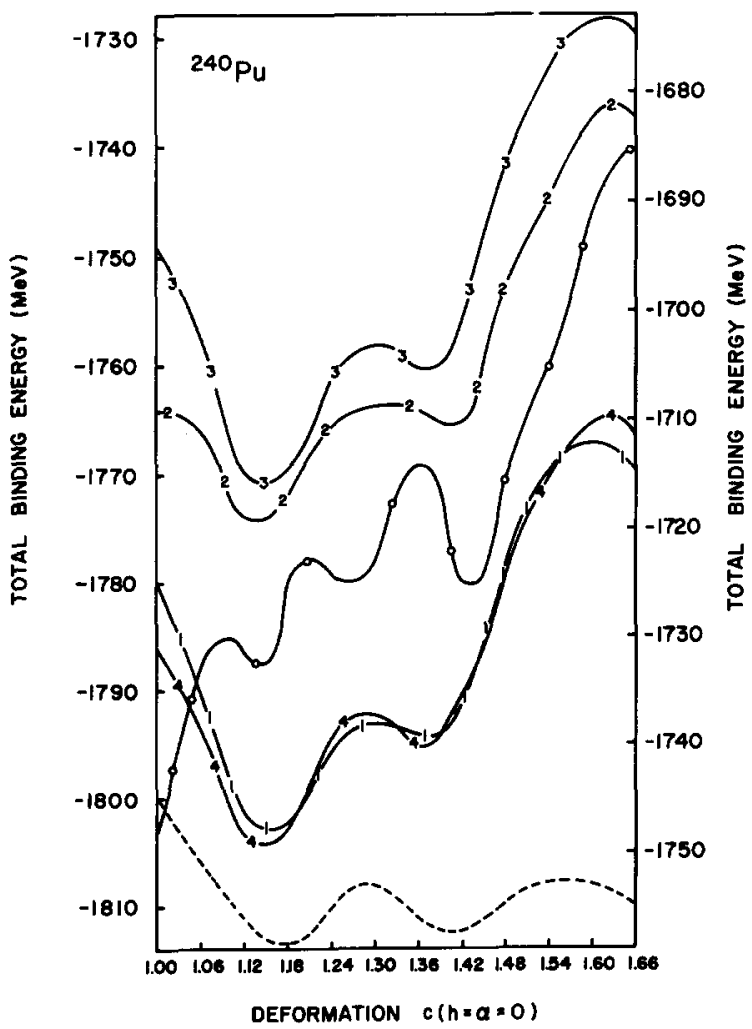

Fig. 1. Deformation energy curves of ${ }^{240} \mathrm{Pu}$ for shapes with $h=\alpha=0$, obtained with the deformed shell model wave functions for various effective interactions: (-1-) SKY 1; (-2-) SKY II; (-3-) SKY III; (-4-) SKY X; (-0-) MDI II. For comparison, the dashed curve (-..-) shows the result obtained with the shell-correction method. For SKY II and MDI II, the scale is on the right. The interaction SKY XP would give a curve which coincides with that for SKY X (4) to within $\approx 1 \mathrm{MeV}$. energy obtained depends on the oscillator constant $\hbar \omega$ which must be explicitly optimized. This is included in our calculations.

We report here some preliminary results of our investigations. Table 1 shows the parameters for the various interactions used in our calculations. Fig. 1 shows the deformation energy curves for $240 \mathrm{Pu}$ obtained with these interactions and also with the shellcorrection method. These curves are calculated for shapes having $h=\alpha=0$ in the $\{c, h, \alpha\}$ parametrization $[3,8]$ which corresponds approximately to the valley region in the deformation energy surface obtained with the pure liquid drop model. As shown in ref. [3], the ground state of ${ }^{240} \mathrm{Pu}$ has a deformation with $h \neq 0$. Right-left asymmetric deformations $(\alpha \neq$ 0 ) are known to lower the second saddle point, and non-axial $\gamma$-deformations affect the inner saddle point. Of course, quantitative predictions can only be obtained after the total binding energy has been minimized with respect to these most important degrees of freedom. However, for a qualitative study of the deformation energy curves, the shapes with $h=\alpha=0$ give a fair approximation and are sufficient for studying the effects of various interactions.

As can be seen from fig. 1, the deformation energy curves obtained from our microscopic calculations with the Skyrme interactions do show a second minimum and two fission barriers at about the same deformations as in the the shell-correction calculations!

$\dagger$ According to the shell-correction calculations the maxima, along $h=\alpha=0$ corresponds to saddle points or fission barriers in the full $\{c, h\}$.space. 
The very different behavior of the deformation energy curve obtained with the Moszkowski interaction is due to its lack of p-wave components.

Since the shell model Hamiltonian we are using does not commute with the total momentum operator and the total angular momentum operator, the deformation energy calculated includes some spurious center-of-mass kinetic energy and rotational energy. As we are only interested in nuclear deformations which conserve both the total momentum and the total angular momentum, these energies should be subtracted. It is found that the center-of-mass kinetic energy changes negligibly with deformations and has approximately the constant value $6 \mathrm{MeV}$. The rotational energy, however, is important and lowers the inner barrier and the isomer minimum by about $1 \sim 1.5 \mathrm{MeV}$ and the outer barrier by about $2 \mathrm{MeV}$, both relative to the ground state.

To test our assumption that the shell model wave functions are sufficiently close to the self-consistent wave functions, we have carried out the constrained Hartree-fock calculations for the lighter nucleus ${ }^{168} \mathrm{Yb}$, using the shell model wave functions for shapes

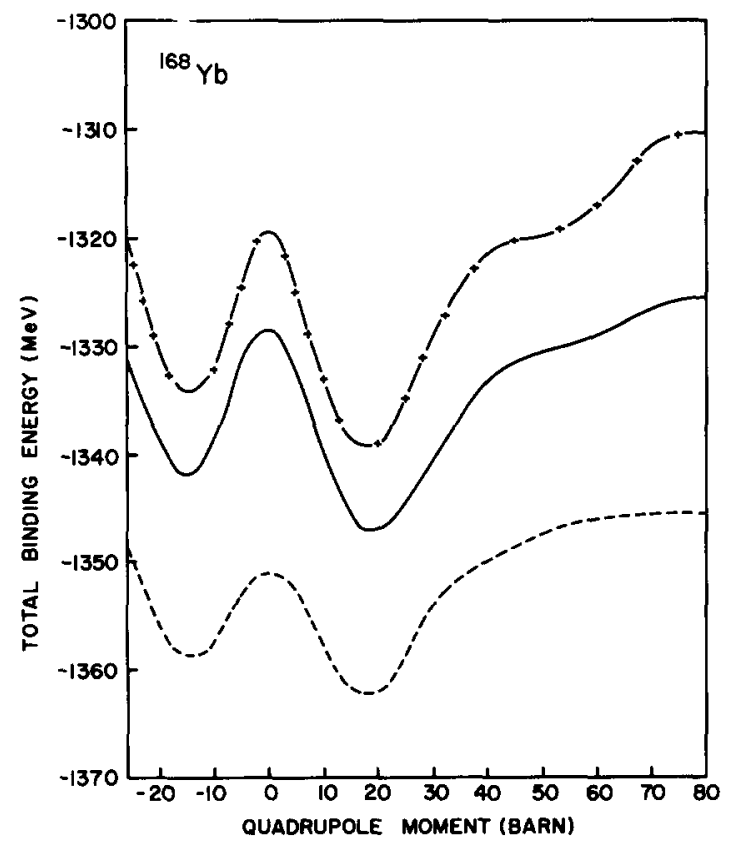

Fig. 2. Deformation energy curves of ${ }^{168} \mathrm{Yb}$ : (-+-) from the deformed shell model wave functions (along $h=\alpha=0$ ) using the interaction SKY XP; (-) self-consistent CHF solution for the same interaction; (-.-) result of the shell-correction method. The quadrupole moment is defined as $Q=\Sigma_{i}\left\langle P_{2}\left(r_{i}\right)\right\rangle$. having $h=\alpha=0$ as the input. From the result shown in fig. 2, we see that the two deformation energy curves agree rather well, considering the fact that we should, in principle, also study the shapes with $h \neq 0$ in our shell model calculations. Furthermore, the ground state quadrupole moments are very close to each other. The total binding energies of the ground state in the two cases differ only by $\sim 8 \mathrm{MeV}$ compared to the total value of $\sim 1350 \mathrm{MeV}$, a result which seems surprisingly good to us and is also confirmed for many other nuclei throughout the periodic table.

However, the barriers in the deformation energy curves obtained either with the shell model wave functions or using the full CHF method [14] are too high as compared to both the experiment and the results from the shell-correction method. Partly this is due to truncation errors at large deformations. Another reason is the too large surface coefficients inherent in the Skyrme interactions [10]. Since all the interactions used here are fitted only to the ground state properties of spherical nuclei, it is likely that better sets of parameters can be found by explicitly fitting to the fission barrier heights and isomer state energies of fissioning nuclei. Our method provides a good tool for this study which is being pursued.

A more detailed discussion of the method and extended results will be presented in a forthcoming publication.

We are indebted to D. Vautherin and coworkers for letting us use their CHF code. One of us (CMK) is very grateful to Professors T.T.S. Kuo and C.W. Wong for many helpful discussions.

\section{References}

[1] H. Flocard, P. Quentin, A.K. Kerman and D. Vautherin, Nucl. Phys. A203 (1973) 433.

[2] V.M. Strutinsky, Nucl. Phys. A95 (1967) 420; A122 (1968) 1

[3] M. Brack et al., Rev. Mod. Phys. 44 (1972) 320.

[4] S.G. Nilsson et al., Nucl. Phys. A131 (1969) 1.

[5] M. Bolsterli, E.O. Fiset, J.R. Nix and J.L. Norton, Phys. Rev. C5 (1972) 1050.

[6] M. Brack and P. Quentin, paper submitted to the Third Intern. Symposium on Chemistry and physics of fission, IAEA 1973, Rochester.

[7] J. Damgaard, H.C. Pauli, V.V. Pashkevich and V.M. Strutinsky, Nucl. Phys. A135 (1969) 432. 
[8] H.C. Pauli, Phys. Rep. 7 C2 (1973) 36.

[9] D. Vautherin and D.M. Brink, Phys. Rev. C5 (1972) 626.

[10] D. Vautherin, Phys. Rev. C1 (1973) 296.

[11] S.A. Moszkowski, Phys. Rev. C2 (1970) 402.

[12] J.W. Ehlers and S.A. Moszkowski, Phys. Rev. C6 (1972) 217 .
[13] J.W. Negele and D. Vautherin, Phys. Rev. C5 (1972) 1472.

[14] P. Quentin et al., preprint "La Toussiere", France, 1973.

[15] H. Flocard, P. Quentin, private communication.

[16] K.F. Liu, private communication 